\title{
Naming Persons in Video: Using the Weak Supervision of Textual Stories
}

\author{
Phi The Pham ${ }^{\mathrm{a}}$, Koen Deschacht ${ }^{\mathrm{b}}$, Tinne Tuytelaars ${ }^{\mathrm{c}}$, Marie-Francine \\ Moens $^{\mathrm{a}}$ \\ ${ }^{a}$ Computer Science Department, KU Leuven, Celestijnenlaan 200A, B-3001 Leuven, \\ Belgium \\ ${ }^{b}$ CULT, USA \\ ${ }^{c}$ KU Leuven, ESAT-PSI, iMinds, Kasteelpark Arenberg 10, B-3001 Leuven, Belgium
}

\begin{abstract}
In this paper, we focus on the problem of automated video annotation. We report on the application of naming faces in soap series by using the weak supervision of narrative texts that describe the events in the video and that are drafted by fans. Several unsupervised methods that operate without any manual labeling of exemplar faces, and methods that use a limited number of labeled exemplars are presented and evaluated. All methods exploit the multiple co-occurrences between faces shown in the video and names mentioned in the texts to compute the strength of the linking and reinforce this coupling by means of an Expectation Maximization algorithm. We show that the unsupervised methods attain competitive results without any prior human effort. The results show $F 1$ values between $80 \%$ and $86 \%$ for the recognition of the face-name pairs without any human supervision. These figures rise only slightly when a number of faces were manually labeled beforehand. The study gives insights in the benefits and bottlenecks of the proposed approaches, and an error analysis results in guidelines for the choice of a certain technique.
\end{abstract}

Keywords: Cross-media mining, video annotation.

Email addresses: PhiThe.Pham@cs.kuleuven.be (Phi The Pham), koendeschacht@gmail.com (Koen Deschacht), Tinne.Tuytelaars@esat.kuleuven.be (Tinne Tuytelaars), Sien.Moens@cs.kuleuven.be (Marie-Francine Moens) 


\section{Introduction}

Video search and mining rely on effective content descriptors extracted from the different media streams (e.g., images, speech, text). Traditionally these descriptors are manually assigned to the data. Current video capturing devices make video a very popular means of transmission and communication of the data. As a result our video archives grow substantially and a manual indexing with content descriptors becomes unfeasible. Recent crowd sourcing approaches, where the video is freely annotated by persons who are possibly paid for their services such as Youtube video annotation, Project Pad ${ }^{1}$, Vertox $^{2}$, Video Annotation and Reference System ${ }^{3}$, BubblePLY ${ }^{4}$, VideoANT ${ }^{5}$, do not constitute a viable solution. The annotations only capture fractions of the available data and they are often noisy containing spam and junk descriptors. Hence, there is a profound interest in automatically generating metadata to label the video data. Here most of the current approaches index the speech transcriptions of dialogues, news broadcasts or other spoken explanations apart from the subtitles if present. Classical word based indices are built from these textual sources, but are often incomplete with regard to the content covered. Automatically extracting content descriptions from the visual data has the disadvantage that we need many labelled examples to train effective classifiers. In a setting where the content is changing rapidly (e.g., the persons and places appearing in the news, or soap series featuring guest actors), this solution again is unsatisfactory. Few research is currently devoted to combining different sources of evidence when automatically labeling video data. For instance, both the textual and visual content can be examined. Evidence found in both media can be combined and eventually may lead to visual detectors that are capable of labeling image data without the presence of related texts. This is also the path that we pursue in this paper. In contrast to previous work where we have aligned faces and names of images and their captions, in this work we focus on aligning faces and names in video. The problem of naming faces in video is challenging because there are often many faces in one image frame or set of frames, and many

\footnotetext{
${ }^{1}$ http://projectpad.northwestern.edu/ppad2

${ }^{2}$ http://digitalhistory.concordia.ca/vertov/

${ }^{3}$ http://vars.sourceforge.net/

${ }^{4}$ http://www.bubbleply.com/default.htm

${ }^{5}$ http://ant.umn.edu/
} 
names in the transcripts of the video. Another difficulty is that the time at which people are named and the time at which the corresponding faces are shown in the image can diverge. The detection and description of the faces in the video frames are also challenging under uncontrolled conditions, due to the wide variability in face appearance - especially because of changes in pose, illumination conditions, facial expressions, and partial occlusions. The image quality when dealing with videos is also significantly lower.

More specifically, we explore several novel methods for labeling persons that appear in video data. We detect faces in the video and assign the most probable name among the names found in associated natural language texts. In particular, we use the weak supervision of narrative texts that describe the events in the video and that are drafted by fans. Several unsupervised methods that operate without any manual labeling of exemplar faces, and methods that use a limited number of labeled exemplars are presented and evaluated. The unsupervised methods are valuable in situations where the number of faces (or names) of a same person is abundant in the frames under investigation, promoting the disambiguation capabilities through redundancy. The supervised methods that rely on user feedback for the labeling are needed when the number of appearances of a person is limited or the face detections are less reliable [1]. We investigate different methods to compute the probability that a name is assigned to a face, which is reinforced through the redundant co-occurence of the concerned name and face patterns. This reinforcement is implemented with an iterative Expectation Maximization (EM) algorithm, which is a method for finding maximum likelihood or maximum a posteriori (MAP) estimates of parameters in statistical models, where the model depends on unobserved latent variables. The method alternates between performing an expectation (E) step, which computes the expectation of the log-likelihood evaluated using the current estimate for the latent variables, and a maximization (M) step, which computes parameters maximizing the expected log-likelihood found in the E step. The methods differ in the initialization of the probabilities of name-face pairing and in the computation of the EM where three different likelihood functions are maximized.

We evaluate our methods on two episodes of the Buffy the Vampire Slayer soap. The results show $F 1$ values between $80 \%$ and $86 \%$ for recognition of face-name pairs without any human supervision. These figures rise only slightly when a number of faces were manually labeled beforehand. All results exploit the weak supervision provided by the textual stories and the person names found in these stories. The study gives insights in the benefits 
and bottlenecks of the proposed approaches, and an error analysis results in guidelines for the choice of a certain technique. Overall, our alignment methods overcome the limited quality of the initial timing alignment of the names and faces and the face detection and description by exploiting the repeated occurrence of names and faces over the video frames and introducing the clothing information, a more reliable source of person appearance. The results are better than state-of-the-art work on the same dataset [2]. The contributions of this paper are the application and comparison of different face labeling techniques that combine evidences obtained from the visual and textual data and that benefit from this combination. Our unsupervised face naming methods can be easily extended to deal with user feedbacks for labeling seed examples. We also introduce an active learning approach to effectively help the users to label example faces.

The remainder of this paper is organized as follows. In the next section we describe related work. Then, in section 2 , we describe and formally define the task of naming faces in video, and discuss the workflow of the system used. In section 4 we discuss the video data preprocessing, i.e., the detection and clustering of the person names in the transcripts, the face detection and tracking in the video frames, and the temporal alignment of video frames and textual transcripts. Section 5 discusses the likelihood functions and the training with the EM algorithm. It also describes the initialization of the probabilities in the EM algorithm and includes unsupervised methods and methods that rely on a small amount of manually labeled exemplar faces which are selected by an active learning approach. In section 6 the experiments are described and the results are given and discussed including the error analysis. Section 7 concludes the paper.

\section{Related work}

Several researchers have looked into the problem of linking names and faces in video, be it with a manual initialization (e.g. $[3,4,5]$ ) or fully automatic based on temporal co-occurrence of names and faces (e.g. [6, 2]). The initial information of names and faces correspondence is then used as a training set for a regular face classification process [3], a multiple instance learning process [4], or in a nearest-neighbor framework [5, 2].

Name-It [6] is one of the pioneer systems proposing the techniques to associate names and faces in videos. In this system, based on the initial information of name and face temporal co-occurrence, a heuristic method 
is applied to select the name-face pairs with the most significant temporal correspondence. This system is restricted to the temporal alignment of the names and the faces.

In their supervised face naming system, Yang et al. [3] first detect the person names from the video transcripts. Then they extract multimodal features from the video that would best identify the people, e.g., transcript clues (when a person says "I'm Peter Jennings. Have a good night", the name "Peter Jennings" is the name of the person in the shot), video OCR text which contains the name of the current person, speaker identity from the video speech, etc. Several constraints are also investigated, e.g., visual similarity (if a person appears several times in a news story, the shots that he/she appears are likely to have the same background, clothes, illuminations, and camera position), and speaker identity (normally, the speaker identity from the monologue audio belongs to the speaker in the corresponding video shot). Finally, the video shots with their extracted features, constraints and the manual annotated names are used to train a Support Vector Machine (SVM), which in the experiments shows interesting person labeling results for the video shots. Instead of using this discriminative model, we propose a generative probabilistic approach in this paper.

Further improving their work by tackling the problem of using heavy human annotation for training examples for the SVM, Yang et al. propose a Multiple Instance Learning approach [4] to label faces in the video. There, each face is associated with several name candidates extracted from the same news story, from which only one name is assumed to be correct. Although these authors use a classifier trained from both correctly and incorrectly labeled examples, we improve the initial classification with an ExpectationMaximization algorithm.

Also working on the problem of face labeling in "Buffy the Vampire Slayer" soap videos, Everingham et al. [2] use an exemplar based classification method. There, they collect the face tracks with only one candidate name as exemplars. Based on these exemplars, they propagate the face labels to the nearest unlabeled neighbour face track. They combine facial and clothing similarity in a face track similarity function. To further improve the quality of the exemplar selection process, they also use speaker detection to assign the mentioned name to the face that is speaking in the video. Based on the exemplar-based classification technique by Everingham, Ramanan et al. [5] propose a nearest neighbour technique to label faces from an archive of a large amount of videos. Besides the facial features, they also use hair 
and torso features to track people in the shot-scale video fragments. In the inter-shot scale, they include clothing information to cluster the tracks. For person clustering across the videos, after a parameter learning process, they show that torso color and facial color are not predictive of the identity in a long time scale. In our experiments we compare the results of our method with those of [2].

Based on a large news photo with caption collection, Ozkan and Duygulu [7] propose a graph based method. They first use the queried name to collect a set of associated faces. Then a weighted graph is built where the nodes are the associated faces and the edges are their similarities. Finally, they find the densest component - a set of highly connected nodes of the graph, and consider this set of nodes to correspond to the faces of the queried person. This method has later been refined by Guillaumin et al. [8], who also extend the method to deal with the multi-person naming problem and include a query expansion step [9]. To annotate a photo collection of different users taken over a period of 8 months, Cao et al. [10] first obtain a set of high confidence annotation labels using a supervised classifier. Then they propagate these labels to the remaining photos by a probabilistic label propagation method, which uses timing, location and visual proximity information. Grauman and Darrell [11] solve a different problem (unsupervised object discovery), yet use a somewhat similar technique: a graph is constructed, with all images as vertices and edge weights based on image similarity (number of feature matches). Then they use normalized cuts to partition the graph in different clusters ("objects"). All these methods use faces (or objects) as vertices of the graph.

Probably most similar to our unsupervised face naming approach is the work of Berg et al. $[12,13]$. They study the problem of linking the faces appearing in an image with the names mentioned in an associated text. They represent the faces in a face appearance space based on kernelPCA and cluster them using a Gaussian mixture model, where each component is assumed to correspond to a specific person. The parameters of the Gaussian mixture model are learnt together with the most probable assignments between detected faces and detected names based on an Expectation Maximization algorithm. The same framework has also been applied in the context of automatic image annotation (i.e. linking image regions with keywords) by Duygulu et al. [14]. It should be noted though that the work of $[7,10,11,12,13]$ regard automated annotation of static images, not of video as we do.

In this paper, we extend our previous work on unsupervised face naming 
in images [15] as follows. First, the face naming methods are adapted to work on video data, instead of image-text data. Second, we use different initialization methods for the Expectation - Maximization alignment algorithm, in which we focus on face feature optimization for video data. We also introduce an active learning approach to allow for user feedback to improve the final alignment results. In videos, although we deal on average with less faces to be labeled per image frame, we meet more difficulties doing face naming than in an image-text dataset (e.g. the "Labeled Faces in the Wild" dataset $[12,13,15])$. First, we see more low quality video frames where the detected faces are more varied in lighting, pose and expression. Second, the text supervision for the alignment is incomplete as in many cases there is no relevant text description from the transcripts.

For the supervised classification/alignment task, learning to select a valuable set of labeled training examples is clearly one of the research priorities $[16,17]$. Active learning is selected as a mechanism for user feedback in an environment where the data is huge and/or diverse while annotation by humans is limited to labeling only a part of them. Hence, the selected data to label should be representative and informative [17]. To guarantee these two properties especially in the area of multimedia processing and retrieval, the manually selected data should be uncertain enough to be informative and diverse enough to be representative. To calculate the uncertainty of a data point, $[16,17]$ use the distance from the clusters centers to the data point. For the diversity, the data point should lie far enough from the set of already selected data points. Our work adapts the active learning method from [16] and the experimental results show that this active learning method helps to improve the final face labeling performance. 


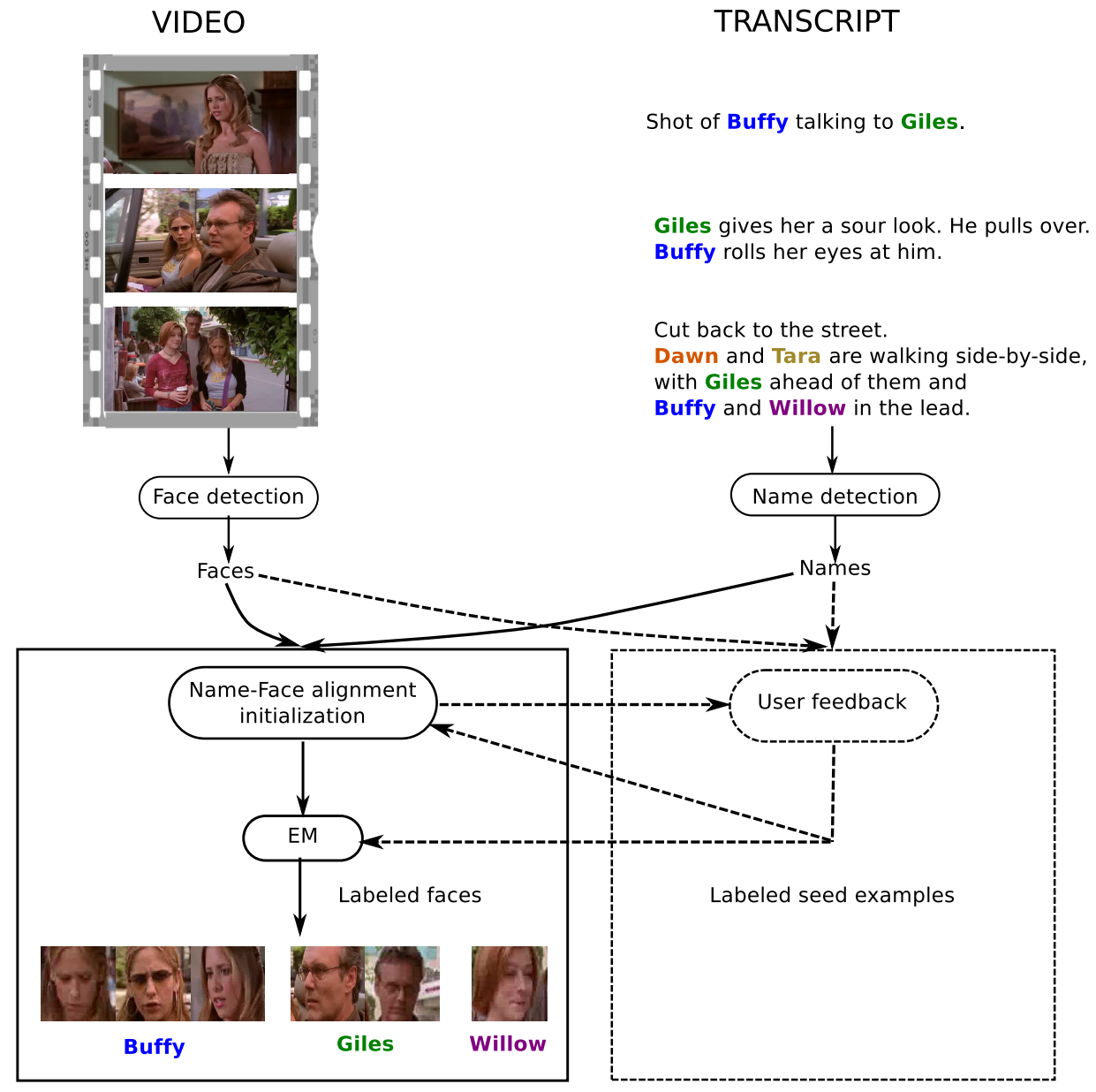

Names and faces alignment Active learning for user feedback

Figure 1: High level overview of the face labeling method. 


\section{Task definition and architecture of the system}

The task is to label each of the $|F|$ faces found in a video episode of Buffy the Vampire Slayer with one of the $|N|$ names found in the transcripts of the video or with the "null" name. Several faces are shown in the video that are never named in the texts. These should be aligned with the null name. Likewise, several names are mentioned in the transcript that do not necessarily occur in the corresponding frames. They should be assigned to the null face. In the transcripts fans account on what happens in the video. Not all shots of the video have comprehensive transcripts, but we assume that the visual and textual data contain sufficient evidence to align faces with names, and to name similar faces in cases that no transcripts are available.

Figure 1 shows the architecture of our face labeling system. In a first step the video and textual data are preprocessed and transcripts are roughly aligned with the textual data. In a next step the probabilities of the face naming are initialized. For this step, two different approaches are proposed. The first approach relies on the unsupervised clustering of names and of faces. The second approach takes into account the co-occurrence of a name with the new face descriptor features. In a next step we use an EM algorithm to improve the initially obtained probabilities by reinforcing the multiple cooccurrences of the names and faces. Three different likelihood functions are maximized. A first one only takes into account the co-occurrence of a name and a face in the transcript. The second one adds evidence on the picturenedness and namedness [15], which respectively refers to the probability that a name in the text is pictured and that a face in the video frame is named. The third likelihood function takes into account the co-occurrence of a name with the face descriptor features. This stepwise approach allows us to evaluate the performance at different stages of the process. We also investigate the effect of taking into account labeled seed examples both in the initialization step and in the EM step, allowing us to compare the performance of an unsupervised versus a supervised approach. The seed examples to be labeled are randomly chosen or are selected with an active learning procedure.

\section{Video data preprocessing}

\subsection{Temporal alignment of transcript and subtitle}

In the "Buffy the Vampire Slayer" episodes, person names are detected from two sources of textual information: the subtitles included in the DVD 


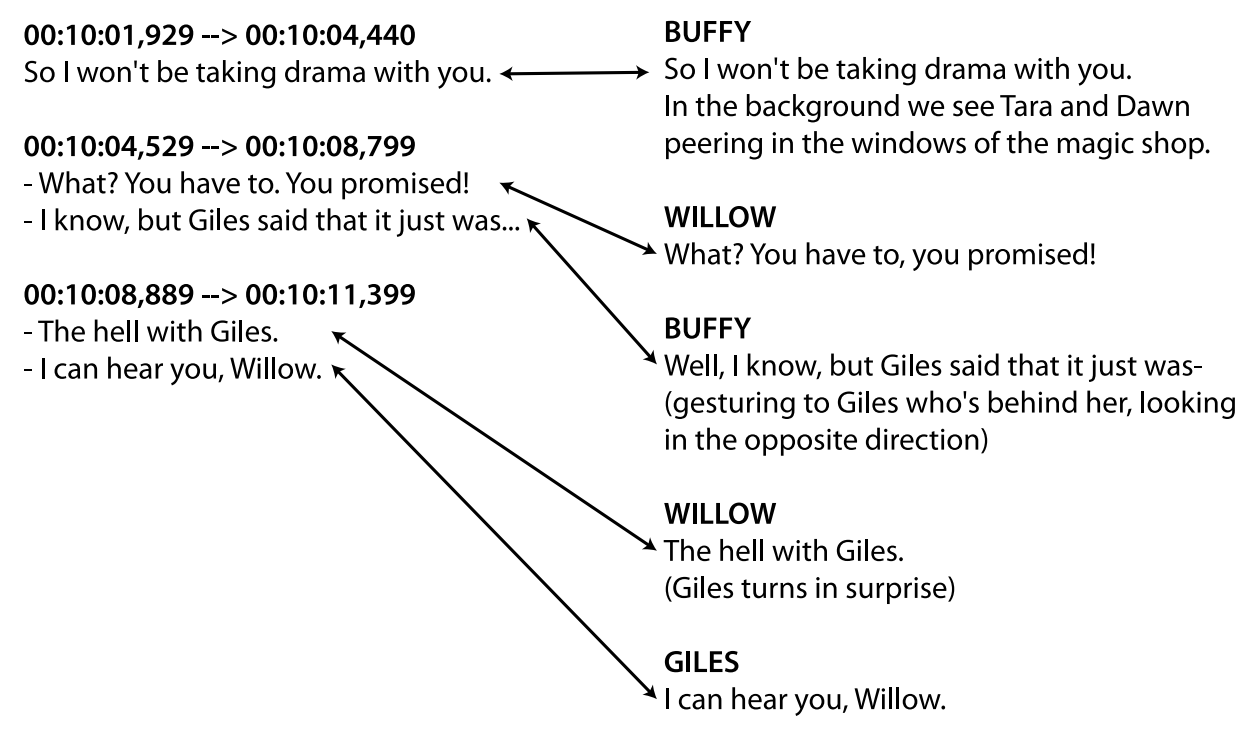

Figure 2: The alignment of transcript (right) and subtitle (left).

and the scripts obtained from a fan website, also used in [2]. The scripts contain the information of who is speaking, what the spoken lines are, and partial descriptions of the actions of the characters. But no timing information is provided. The subtitles contain the information of when the spoken lines occur. A dynamic time warping algorithm [2] is used to align the scripts and subtitles to obtain information of who is speaking/doing what and when. An illustration of the time warping algorithm is shown in figure 2 .

\subsection{Name extraction and clustering}

We use a named entity recognizer which is based on a maximum entropy classifier from the OpenNLP package ${ }^{6}$. The initial output of our name detection method is the list of the detected names in the scripts and the corresponding range of frames (timing information) during which the names are mentioned. Coreference chains are formed based on the detected names. Then coreference chains of each text are clustered with a hierarchical single link algorithm constrained by a threshold cosine similarity for cluster membership.

\footnotetext{
${ }^{6}$ http://opennlp.sourceforge.net/
} 


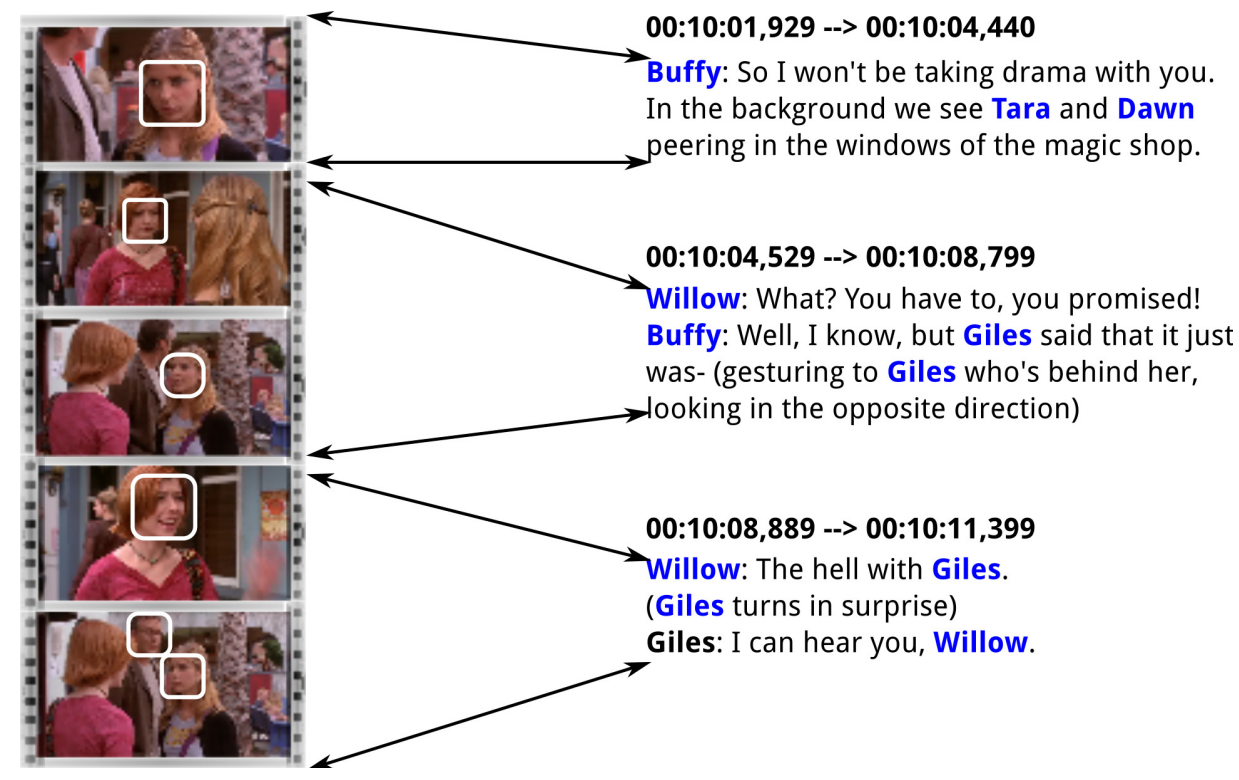

Figure 3: The temporal alignment of names and faces.

\subsection{Face detection and clustering}

The faces from the video frames are detected using the OpenCV implementation of [18]. Then the SIFT features [19] of the face appearance are extracted by computing descriptors of the local appearance of the face around each of the located facial features (eyes, nose, mouth) [2]. These faces arise from just a few hundred "tracks" of a particular character each in a single shot [2]. Applying a face-tracking method reduces the amount of data to process and allows us to select the best faces in each face track to help improve the quality of face comparison and classification. We use the Kanade - Lucas - Tomasi [20] method to track faces using similar point tracks over frames. The faces are clustered by a hierarchical agglomerative clustering algorithm and a cosine similarity metric, more specifically Group Average Clustering (GAC) with a predefined threshold of cluster membership similarity.

\subsection{Temporal alignment of names and faces}

Finally, given the transcripts with the timing information obtained from the transcript alignment process, we temporally align the detected faces from 
the video frames and the extracted names from the transcripts as follows: Given the temporal segment of the transcript, if a face track is temporally overlapping with this transcript segment, all the member faces of the face track will inherit all the candidate names from the transcript segment. An example of the temporal alignment of names and faces is shown in figure 3. This temporal alignment process is essential to our later unsupervised names and faces alignment method as it defines the names that are candidates to be assigned to the faces shown. Here, the temporal segment of the transcript is obtained from the temporal segment of the subtitle which is aligned to the transcript via the transcript alignment algorithm.

\section{Computing the likelihood of the face naming}

Given a video with automatically detected faces, a transcript with automatically detected names, and an approximate temporal alignment of the transcript with the video, we consider all possible alignments of the detected faces with the detected names in each time-delimited segment. Figure 4 shows the 7 possible alignment schemes that should be considered for an example video frame with two detected names and two detected faces.

For each frame, we want to estimate the probability of every alignment scheme. Since faces and names typically occur multiple times in the video, the algorithm can learn automatically what faces are more likely to be associated with a certain name and vice versa.

We consider three different methods. All of them start from the likelihood of the possible alignments between names and faces. Potential alignments are initialized with an estimated probability, in our case the joint probability $P(n, f)$ for each name $n$ and face $f$, in the computations below often split into $P(f \mid n)$ and $P(n)$. An expectation maximization approach is used to update the likelihood of $P(n, f)$. In each frame-text pair $s_{i}$, given $\left|N_{i}\right|$ names including the null name, there are many possible alignment schemes $a_{j}$ to assign $\left|F_{i}\right|$ faces and a null face to these names (see figure 4), from which we have to choose the best one. The constraint for each alignment scheme is that a face must be assigned only to one name, while the null face can be assigned to any name and the null name to any face.

In the following sections we first discuss the different initialization meth-

ods for estimating $P(f \mid n)$ that are unsupervised or that take into account a limited amount of manually annotated exemplar faces. Then we discuss how 

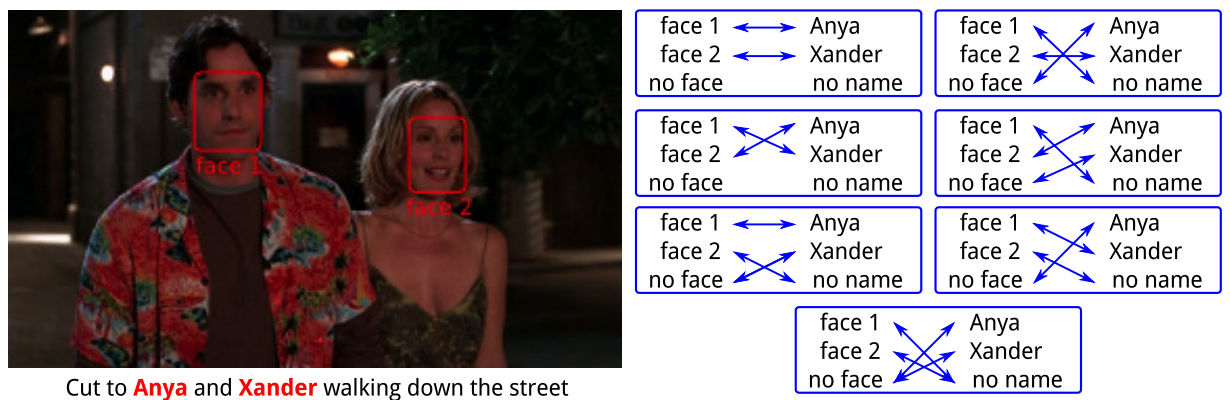

Figure 4: Different alignments for an example frame with two detected faces and two detected names in the associated transcript.

these estimations can be improved by using different likelihood functions and a corresponding EM algorithm.

\subsection{Initialization}

To make EM converge to global optimum, good initial values for the distributions $P(f \mid n)$ are essential. We consider three different options: Initializing $P(f \mid n)$ based on initial clustering, initializing $P(f \mid n)$ based on a Naive Bayes classifier trained with noisy alignments and initializing $P(f \mid n)$ based on seed examples.

\subsubsection{Initializing $P(f \mid n)$ based on initial clustering}

As described in section 4, we have identified clusters of similar names and clusters of similar faces that ideally each represent one person name and one person face respectively, although in practice, they will be mixtures of different people, especially for the faces. We adapt the method proposed by Mori et al. [21], where frames inherit all the person names of the text that co-occur with a face. The $P(f \mid n)$ distributions are estimated by counting the relative frequencies of the face clusters inherited by a given name. This is somewhat reminiscent of the approach taken by [7], who find the densest component in a similarity graph of names co-occurring with a particular query name - but note that we cluster all faces simultaneously.

We denote $\operatorname{sim}_{f_{i} f_{j}}$ as the similarity between two faces $f_{i}$ and $f_{j}$ using the cosine similarity metric (see section 4). In videos, computing face similarity is challenging because of different size, expression, pose and lighting. As exploited in [2], incorporating the appearance of the clothing helps improving the quality of the face similarity functions. 


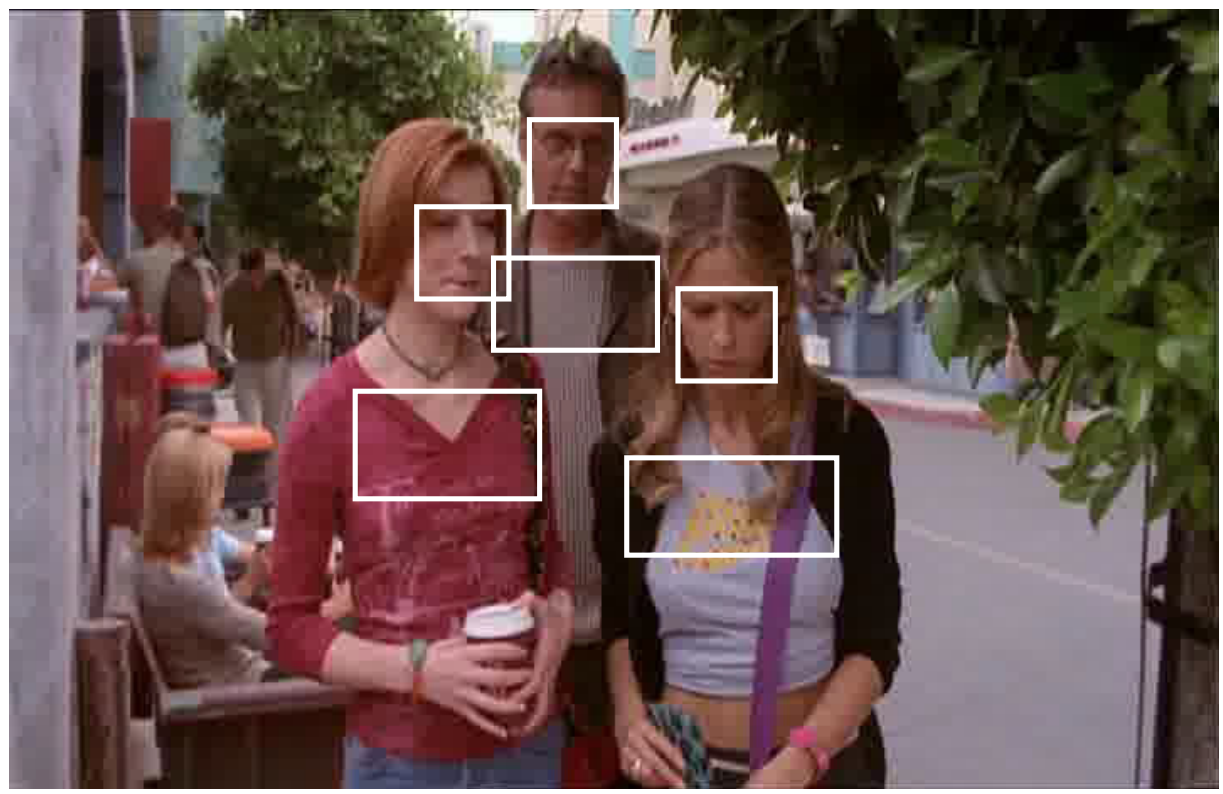

Figure 5: The clothing boxes are detected relative to the size and the position of the corresponding faces .

The clothing box is predicted relatively to the size and the position of the corresponding face. In our experiments, the width of the clothing box is twice the size of the face, the length of the clothing box is the same as the size of the face and the position of the clothing box is under the face at a distance equal to the size of the face (see figure 5). Then the description of the clothing box is presented by the HSV color histogram. We compute clothing box similarity:

$$
\operatorname{sim}_{c_{i} c_{j}}=\exp \left(-\frac{d_{c_{i} c_{j}}^{2}}{\sigma_{c}^{2}}\right)=\exp \left(-\frac{\sum_{k=1}^{M}\left(c_{i}^{k}-c_{j}^{k}\right)^{2}}{\sigma_{c}^{2}}\right)
$$

where $M$ is the number of bins in the histogram and $\sigma_{c}$ is the maximum distance between all clothing boxes.

Given two persons $e_{i}$ and $e_{j}$ with corresponding faces and clothing $\left(f_{i}, c_{i}\right)$ and $\left(f_{j}, c_{j}\right)$, the similarity between them is:

$$
\operatorname{sim}_{e_{i} e_{j}}=\gamma \operatorname{sim}_{f_{i} f_{j}}+(1-\gamma) \operatorname{sim}_{c_{i} c_{j}}
$$


Here, we set $\gamma$ to 0.5 .

\subsubsection{Initializing $P(f \mid n)$ based on a Naive Bayes classifier trained with noisy alignments}

The use of clusters of faces as described in the previous section has some advantages, such as leading to simple likelihood functions that can be constructed in different ways and a high level of symmetry between the visual and textual modality. But clustering also has some disadvantages, of which the most important one is that the clustering itself is non-trivial: in our case we have videos of 25.000 faces and the clustering requires that the similarity between all pairs of faces needs to be computed. Furthermore, it can never be fully avoided that some faces of different persons are put together in the same cluster.

Instead of clustering the names and faces of an episode, we can train a classifier in a weakly supervised manner, based on the information of the time warped segments, using the mentioned names as an automatically assigned label to each face. That is, we use the initial $P\left(n_{k} \mid f_{t}\right)$ distributions in a Naive Bayes classifier. Then, we use this classifier to estimate the probability of a face given a name based on the features of the faces.

More specifically, every face $i$ is described by a feature vector $\vec{f}_{i}=$ $\left\{f_{i}^{1}, \ldots, f_{i}^{D}\right\}$ of continuous numbers (a concatenation of different SIFT descriptors). In previous research, a classifier is built by assuming that the features derive from a normal distribution, and for every face a normal distribution was chosen that optimizes the likelihood of the training data [13]. However, initial experiments showed that this approach performed badly on our data. Therefore we chose a different approach, where we discretize the individual features and use these discretized features to learn a simple Naive Bayes classifier.

For every feature index $j$ we discretize the features $f_{i}^{j}$ of all faces $f_{i}$ by collecting all values of these features in a sorted collection $C=\left\{f_{1}^{j}, \ldots, f_{|F|}^{j}\right\}$, where $|F|$ is the total number of faces in the dataset. $C$ is divided into $\mathrm{B}$ number of bins. Let $b$ be the index of bins ranging from 1 to $B$, each $f_{i}^{j}$ in bin $b$ recieves the value $g_{i}^{j}=b$.

After this discretization, every face $f_{i}$ is thus described by a feature vector $\overrightarrow{g_{i}}=\left\{g_{i}^{1}, \ldots, g_{i}^{D}\right\}$ of length $D$ where every feature $g_{i}^{j} \in\{1, \ldots, B\}$. The application of the Naive Bayes classifier then generates for every face $f_{i}$ : 


$$
P\left(n_{k} \mid f_{i}\right)=\frac{P\left(n_{k}\right) \prod_{j=1}^{D} P\left(g_{i}^{j} \mid n_{k}\right)}{P\left(f_{i}\right)}
$$

Then the probability of a face $f_{i}$, given a name $n_{k}$ is computed as:

$$
P\left(f_{i} \mid n_{k}\right)=\frac{P\left(n_{k} \mid f_{i}\right) P\left(f_{i}\right)}{P\left(n_{k}\right)}
$$

The estimation of $P\left(f_{i}\right)$ and $P\left(n_{k}\right)$ is as follows. Given the name clusters obtained in section $4, P\left(n_{k}\right)$ is computed by maximum likelihood estimation of the occurrence of a name among all names in the collection. Given the face clusters obtained from the Naive Bayes classifier, $P\left(f_{i}\right)$ is computed by maximum likelihood estimation of the occurrence of a face among all faces in the collection.

\subsubsection{Initializing $P(f \mid n)$ based on seed examples}

The above initialization methods also allow to integrate evidence obtained from labeled seed examples. In that case the name-face pairs that make up the seed exemplars receive an alignment probability of 1 and other nameface pairs that involve only a name or a face that belongs to the labeled positive examples receive a probability of zero. More formally, given the set of labeled faces $F_{l}$ a name-face pair $(f, n)$ with $f \in F_{l}$ and the label of $f$ being $n$, the probability $P(f \mid n)$ receives the value of 1 . Other pairs $\left(f, n_{o}\right)$ or $\left(f_{o}, n\right)$ with $f<>f_{o}$ and $n<>n_{o}$ receive the probability of 0 . All other name-face pairs are computed as described in sections 5.1.1 or 5.1.2. These probability assignments apply both when using face clusters and when using face feature vectors. The seed examples are obtained by two approaches: random selection or by using an active learning approach where the machine intelligently chooses example faces to be labeled.

We choose a face naming approach, not the other way around (adding a face to a name) because: 1) The face naming approach is more efficient since the user can easily select a name from a short list to label a face; 2) It is more useful since the process will select the difficult faces to be labeled.

An unlabeled face is valuable to label if the alignment EM is uncertain about its label prediction and if it lies far away from the already labeled faces (diversity). After the initialization process finishes, a face $f_{\tau(\alpha)}$ is assigned to a name $n_{\alpha}$ with the probability $P\left(f_{\tau(\alpha)} \mid n_{\alpha}\right)$. Here, the lower the $P\left(f_{\tau(\alpha)} \mid n_{\alpha}\right)$ value is, the more uncertain the face label prediction is. We denote this 
$P\left(f_{\tau(\alpha)} \mid n_{\alpha}\right)$ value as $s_{1}(f)$. Additionally, we define the distance between the unlabeled face $f$ and the set of labeled faces $F_{l}$ as follows: $s_{2}(f)=$ $\max _{f_{l} \in F_{l}} \cos \left(f, f_{l}\right)$ with $\cos \left(f, f_{l}\right)$ the cosine similarity between the features of faces $f$ and $f_{l}$. The intuition is that the smaller the value of $s_{2}(f)$ is, the further $f$ lies from the set of labeled faces $F_{l}$. The reason for starting the active learning process after the initialization of the $P\left(f_{\tau(\alpha)} \mid n_{\alpha}\right)$ distribution is that, the manual labeled examples can be used to improve the initial probability estimates and to train all three types of likelihood functions. Thus, this helps saving a significant amount of user annotation time.

The active learning process works as follows: Choose a preset number of seed examples which are not yet labeled (the preset number is discussed in section 6.3) with the lowest combination of $s_{1}(f)$ and $s_{2}(f)$ values one at a time and in a greedy fashion and provide them to the user to label:

$$
f_{s}=\operatorname{argmin}_{f \in F_{u}} \gamma s_{1}(f)+(1-\gamma) s_{2}(f)
$$

In our experiments, we set $\gamma=0.5$.

\subsection{The EM-algorithm}

\subsubsection{Likelihood based on $P(n, f)$}

We define the likelihood of the alignment scheme $a_{j}$ for story $s_{i}$ as follows:

$$
\begin{aligned}
L_{s_{i}, a_{j}}^{(n, f)} & =\prod_{\alpha} P\left(f_{\tau(\alpha)}, n_{\alpha}\right) \\
& =\prod_{\alpha}\left(P\left(f_{\tau(\alpha)} \mid n_{\alpha}\right) P\left(n_{\alpha}\right)\right)
\end{aligned}
$$

where $\alpha$ is the index to the not null names of story $s_{i} ; \tau(\alpha)$ is the index to the faces (including the null face) assigned to these names $\alpha$.

$P\left(n_{\alpha}\right)$ is computed by maximum likelihood estimation of the occurrence of a name among all names in the collection.

The complete log-likelihood of all stories $S$ is:

$$
\sum_{s_{i} \in S} \sum_{a_{j} \in A_{i}} \delta_{i, j} \log \left(L_{s_{i}, a_{j}}^{(n, f)}\right)
$$

The probability of the alignment scheme $a_{j}$ of frame $s_{i}$ is denoted by $\delta_{i, j}$ and is updated with an EM algorithm in two steps: 


\section{E-step}

During the E-step, the likelihood $\delta_{i, j}$ of each alignment scheme $a_{j}$ for frame $i$ is computed as:

$$
\delta_{i, j}=\frac{L_{s_{i}, a_{j}}}{\sum_{a_{l} \in A_{i}} L_{s_{i}, a_{l}}}
$$

2. M-step

In the M-step we update the probability distribution $L_{s_{i}, a_{j}}$ based on the estimated likelihoods $\delta_{i, j}$. During the M-step the parameter $P(f \mid n)$ is recomputed using soft counts:

$$
P(f \mid n)=\frac{\sum_{s_{i} \in S} \sum_{a_{j} \in A_{i}} \delta_{i, j} m\left(a_{j}(n)=f\right)}{\sum_{s_{i} \in S} \sum_{a_{j} \in A_{i}} \delta_{i, j} m\left(n, a_{j}\right)}
$$

where $m\left(a_{j}(n)=f\right)$ is 1 , if a face from the same face cluster $f$ (the result of the face clustering process described in section 4) is assigned to a name of the same name cluster $n$ in the link scheme $a_{j}$, otherwise it is $0 ; m\left(n, a_{j}\right)$ is 1 , if the name $n$ is assigned to a non-null face in $a_{j}$, otherwise it is 0 .

\subsubsection{Likelihood using picturedness and namedness scores}

In the case of a text mentioning several distinct names, we can perform an analysis of the structure of the text to determine the names that are more likely to appear in the associated video. We use the term picturedness [15] to indicate the probability of the not null name index $\alpha$ being present in the video. We compute $P\left(\right.$ pictured $\left._{\alpha} \mid t_{s_{i}}\right)$ based on the salience of the name in the text $t_{s_{i}}$ of the frame-text pair $s_{i}$, assuming salient names are more likely to occur in the image. As in [22] we compute the salience score (ranging from 0 to 1 ) of a noun as a linear combination of the salience of the noun in the discourse and in the syntactic dependency tree of the sentence [23], where we learn the interpolation weights from a small training set. In [22] also a visualness score is computed, but for person names this always equals one. Analogously, not all persons detected in the video are equally likely to be described in the transcript. The largest faces in the image or the faces that appear in the center of the image are more likely to be described by names than small faces in the background. Several appearance cues can be easily obtained from the image: the sizes of the faces, the positions in the images (left, right, center, top, bottom). A visual survey of a limited set of images 
learned that the size of a face is an important indicator of the probability that the face is named in the text. Hence, to estimate the namedness scores $P\left(\right.$ named $\left._{\tau(\alpha)} \mid p_{s_{i}}\right)$ for detected faces in an image $p_{s_{i}}$ of the frame-text pair $s_{i}$, we calculate their areas. The namedness scores are then given by the normalized area values relative to the image size.

We define the likelihood of the alignment scheme $a_{j}$ for story $s_{i}$ as follows:

$$
\begin{aligned}
& L_{s_{i}, a_{j}}^{\left(n^{*}, f^{*}\right)}=\prod_{\alpha, \tau(\alpha) \neq N U L L}\left[P\left(f_{\tau(\alpha)} \mid n_{\alpha}\right) P\left(n_{\alpha}\right)\right. \\
& \left.P\left(\text { pictured }_{\alpha} \mid t_{s_{i}}\right) P\left(\text { named }_{\tau(\alpha)} \mid p_{s_{i}}\right)\right] \\
& \prod_{\alpha, \tau(\alpha)=N U L L}\left[\left(1-P\left(\text { pictured }_{\alpha} \mid t_{s_{i}}\right)\right)\right. \\
& \left.P\left(f_{N U L L} \mid n_{\alpha}\right)\right]
\end{aligned}
$$

\subsubsection{Likelihood function based on the feature vector}

When we have initialized the alignment probabilities based on a Naive Bayes classifier trained with noisy alignments as described in section 4.1.2, we adapt the computation of the likelihood function accordingly.

$$
L_{s_{i}, a_{j}}^{N B}=\prod_{\alpha} P\left(f_{\tau(\alpha)} \mid n_{\alpha}\right)
$$

where $\alpha$ is the index to the not null names of frame $s_{i}$ and $\tau(\alpha)$ is the index to the not null faces assigned to these names.

After estimating the probabilities of the alignments, the probability function of a feature given a name is updated as:

$$
P\left(f_{i}^{j} \mid n_{k}\right)=\frac{\sum_{s_{i} \in S} \sum_{a_{j} \in A_{i}} \sum_{\alpha} \delta_{i, j} m\left(n_{\alpha}=n_{k}, g_{\tau(\alpha)}^{j}=g_{i}^{j}\right)}{\sum_{s_{i} \in S} \sum_{a_{j} \in A_{i}} \delta_{i, j} m\left(n_{\alpha}=n_{k}\right)}
$$

where $m\left(n_{\alpha}=n_{k}, g_{\tau(\alpha)}^{j}=g_{i}^{j}\right)$ is 1 , if name $n_{\alpha}$ equals name $n_{k}$ and feature $g_{\tau(\alpha)}^{j}$ equals feature $g_{i}^{j}$, otherwise it is 0 , and $m\left(n_{\alpha}=n_{k}\right)$ is 1 , if the name $n_{\alpha}$ equals $n_{k}$, otherwise it is 0 .

\subsubsection{Likelihood functions when incorporating labeled seed examples}

When we have access to labeled seed examples, we incorporate the labeled faces $F_{l}$ into the EM process as follows. Only the name-face pairs $(f, n)$ with 
$f$ and $n$ not belonging to $F_{l}$ are updated in the M-step. These probability updates apply both when using face clusters and when using face feature vectors. The reason of clamping the labeled examples into the M-step is that we don't want the valuable labeled information to fade away.

\section{Experiments}

\subsection{Datasets and preprocessing}

We perform and compare our experiments on the two episodes 05-02 and $05-05$ of the TV series "Buffy the Vampire Slayer". Each episode lasts approximately 30 minutes, and is composed of about 60.000 frames.

After the face detection and tracking process, we obtain from each episode an average of 26.000 faces, forming on average 1200 face tracks. The face detection rates (the percentage of correctly detected human faces) in episode 05-02 and 05-05 are $94.48 \%$ and $96.92 \%$, respectively. To reduce the number

of false positives in the face detection, we filter out the detections with a too low confidence value. Finally, to further reduce the processing time, an average of 3 representative faces are selected for each track. These kept faces are first selected by their size (the larger, the better), then by their detection confidence. After reduction, an episode contains on average 1500 faces and 500 face tracks.

For the name detection, we obtain from each episode an average of 12 unique candidate names.

The detailed data preprocessing statistics are shown in table 1 where we report the number of faces and face tracks and person names before and after the data reduction process. Table 2 and figure 6 show the complexity of the preprocessed data in terms of average and standard deviation of the number of faces and names per image frame.

\begin{tabular}{|l||l||l||l||l||l|}
\hline Episode & NDF & NDFT & NRF & NRFT & NDN \\
\hline $05-02$ & 27504 & 1434 & 1581 & 562 & 13 \\
\hline $05-05$ & 25528 & 1028 & 1410 & 493 & 12 \\
\hline
\end{tabular}

Table 1: Data preprocessing statistics. NDF: Number of detected faces; NDFT: number of detected face tracks; NRF: number of faces after data reduction; NRFT: number of face tracks after data reduction; NDN: number of distinct detected names. 


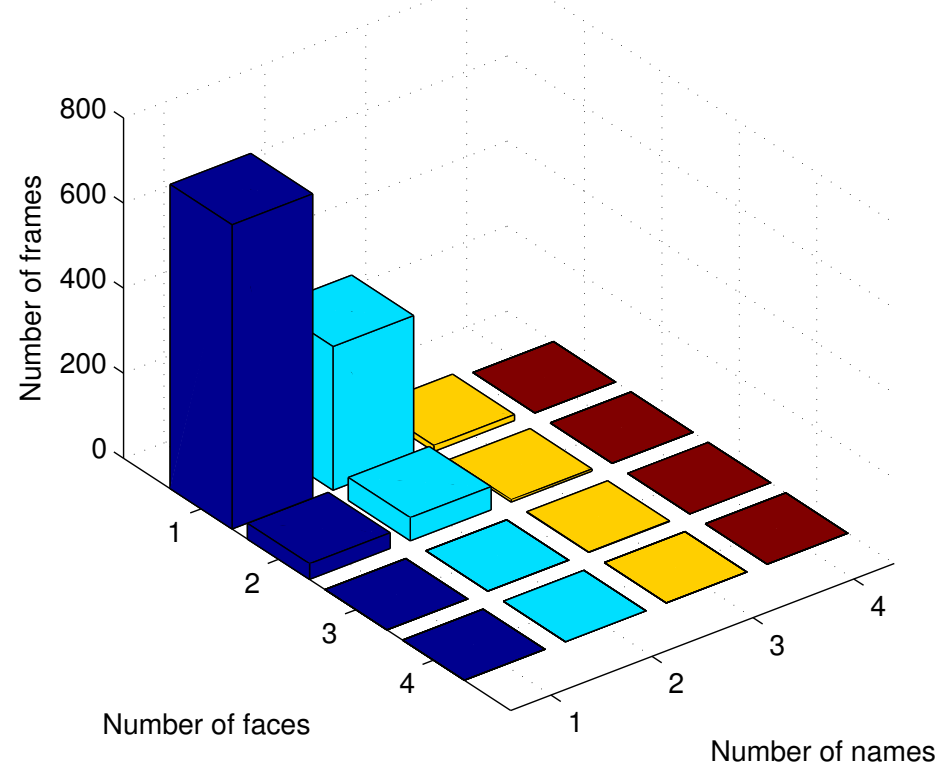

(a) Episode 0502.

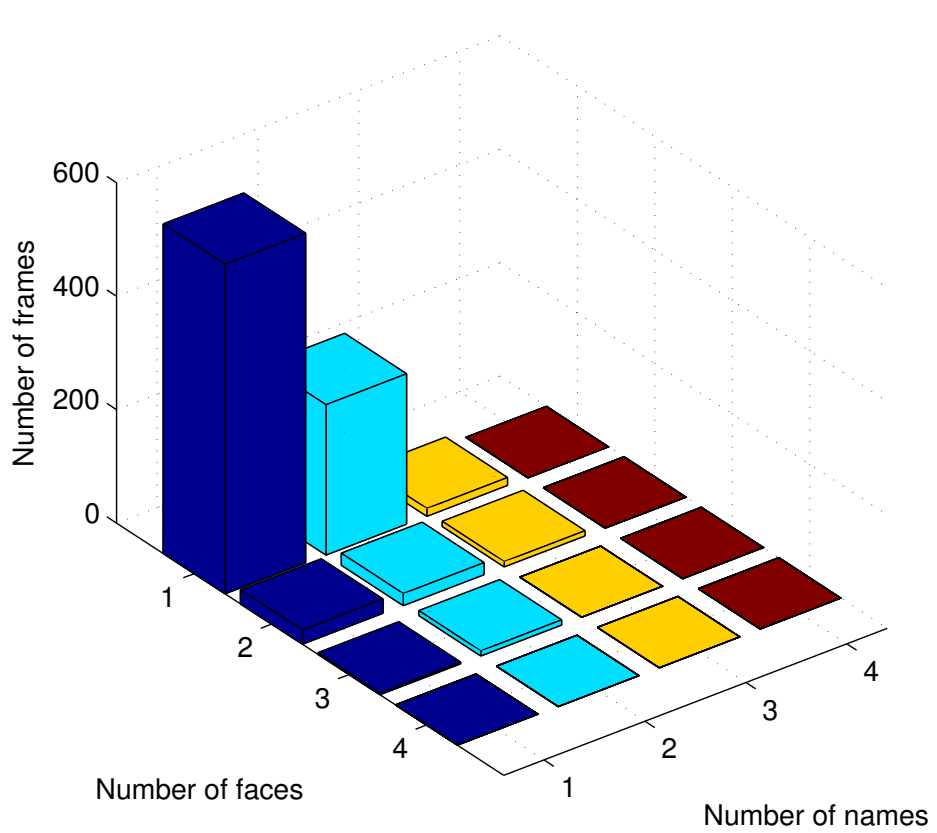

(b) Episode 0505.

Figure 6: Number of names and faces of the Buffy 0502 and 0505 episodes. 


\begin{tabular}{|l||l||l||l||l||}
\hline Episode & AF & DF & AN & DN \\
\hline $05-02$ & 1.084 & 0.008 & 1.371 & 0.015 \\
\hline $05-05$ & 1.085 & 0.010 & 1.367 & 0.017 \\
\hline
\end{tabular}

Table 2: Data complexity estimated per time-aligned video fragment. AF: Average number of faces; DF: Standard deviation of the number of faces; AN: Average number of names; DN: Standard deviation of the number of names.

\subsection{Evaluation specifics}

We consider the ground truth links and compute the recall $R$ and precision $P$ of the automatically generated links. Recall is defined as the percentage of correct links found and precision as the percentage of links found that are correct. We combine recall and precision into the $F_{1}$ measure computed as $\frac{2 R P}{R+P}$. We only consider the links between genuine names and faces (i.e., excluding null name and null face).

For our names and faces alignment methods that rely on labeled seed exemplars, we evaluate all genuine name-face pairs excluding the feedback pairs.

\subsection{Results}

We perform and evaluate our names and faces alignment methods using three likelihood functions: the likelihood function based on $P(n, f)$ denoted as $L^{(n, f)}$ (see equation 6 ), the likelihood function using picturedness and namedness scores denoted as $L^{\left(n^{*}, f^{*}\right)}$ (see equation 10) and the likelihood function based on the feature vector denoted as $L^{N B}$ (see equation 11). With each likelihood function, we perform our experiments in three settings: only using facial appearance (OF), only using clothing appearance (OC) and using the combination of facial and clothing appearances (FC). With user feedback, we repeat the experiments with six different numbers of labeled faces corresponding to $5,10,15,20,25,30$ percent of the faces that are labeled by the user, either selected based on the active learning scheme of section 5.1.3, or chosen randomly.

The results of the unsupervised name-face alignment evaluation by means of precision, recall after applying the EM algorithm with three likelihood types $L^{(n, f)}, L^{\left(n^{*}, f^{*}\right)}$ and $L^{N B}$ are presented in figure 7 . In this figure we see that the likelihood type $L^{N B}$ always outperforms the rest both in term of precision as well as in term of recall. With this scheme we reach $F 1$ scores of $80 \%$ and $86 \%$ on episodes 0502 and 0505 respectively. So we choose this 
likelihood type to further validate our active learning method when selecting exemplars to be labeled by a user. Picturedness and namedness do not seem to help much in this setting (as opposed to images-with-captions as in [15]). In most of the cases the setting using facial and clothing appearances (FC) gives the best results. So we only use this setting for further experiments.

To validate the active learning method, we compare with randomly selected faces. The random face selection process is performed ten (10) times per video episode to guarantee sufficient randomness. The mean and standard deviation of the results of the ten experiments on the randomly selected faces are used to compare against the performance of the experiment on the active learning labeled faces. Figure 9 shows the results of introducing labeled seeds in the face labeling process. Remark that the selection of faces to be labeled based on diversity and uncertainty statistically outperforms the random selection. At the same time, the improvement in precision and recall is rather limited, resulting in F1 scores of $85 \%$ and $88 \%$ for episodes $05-02$ and 05-05 respectively when labeling 10 percent of the faces ${ }^{7}$.

\subsection{Discussion}

In a glance, the alignment methods performance is promising. The incorporation of the clothing information and user feedback helps improving the alignment results. The names extracted from the transcripts provide many, but noisy annotations, and we can conclude from these results that these noisy annotations are informative in order to yield good overall results.

Figure 8 shows a representative set of clusters of faces grouped by their labeled names. From these examples, we see some important properties from the data : 1) Some faces appear frequently (Buffy and Harmony), while others are rare since they appear shortly in the episodes. 2) The lighting, facial expressions, and poses of the faces vary significantly. From our analysis, there are two main factors that prevent the alignment from reaching its utmost performance: the initial timing alignment of the detected names and faces and the quality of the face detection (see section 4). In many cases, we see the timing alignment of the detected faces and their candidate names are not correct. The alignment process compensates this misalignment by using

\footnotetext{
${ }^{7}$ Note that this is excluding the labeled faces in the computation of precision and recall, so can not be compared directly with the $80 \%$ and $86 \%$ mentioned earlier. If we include the labeled faces, we obtain F1 scores of $87 \%$ and $90 \%$, i.e. an increase in performance of $7 \%$ and $4 \%$ respectively.
} 


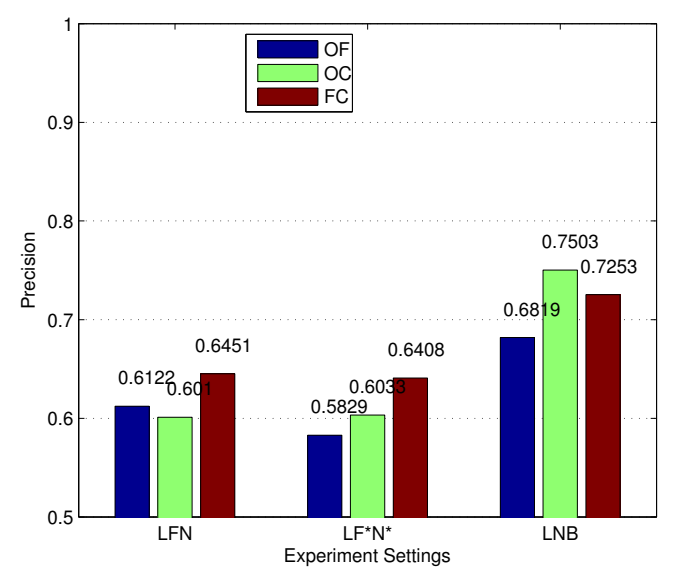

(a) Precision on episode 0502.

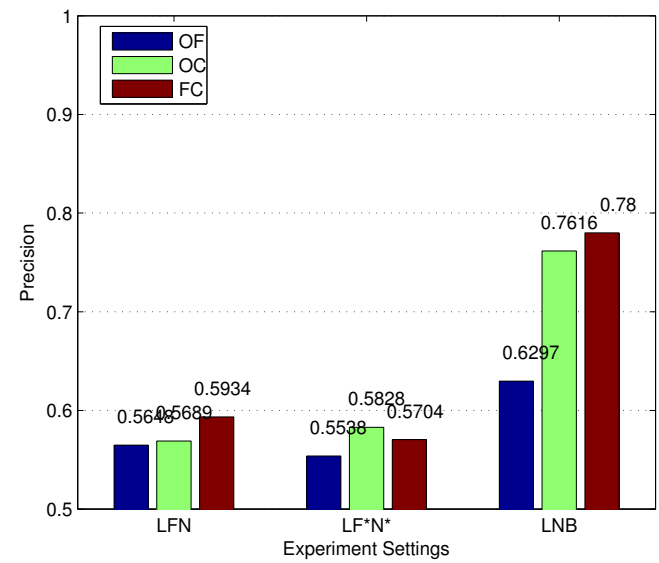

(c) Precision on episode 0505.

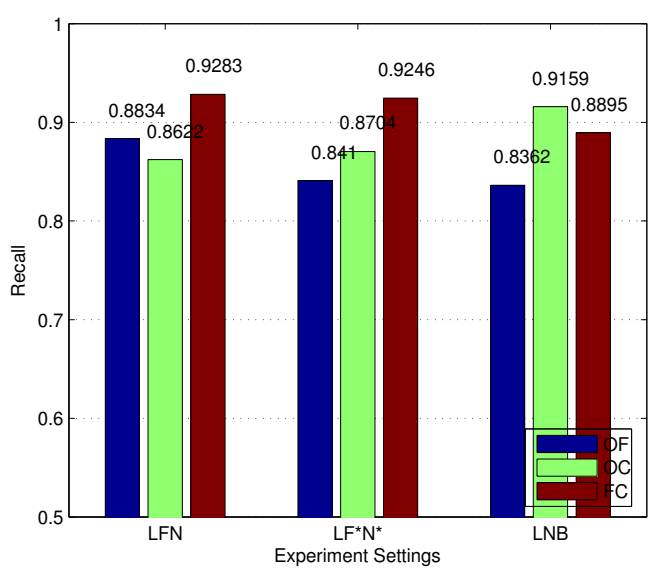

(b) Recall on episode 0502.

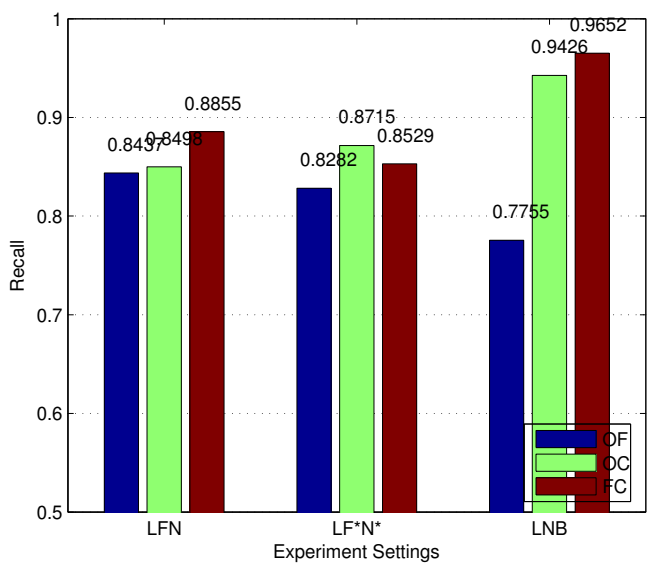

(d) Recall on episode 0505.

Figure 7: Precision/recall values of the unsupervised alignment methods in three settings: using only facial appearance (OF), using only clothing appearance $(\mathrm{OC})$ and using both facial and clothing appearance (FC). 
the face clusters and the repeating appearance of the faces throughout the video with the hope that if in many cases, the faces referring to the same person receive correct candidate names, then the candidate names which are incorrectly attributed by means of time warping can be refused to be assigned to these faces based on the majority of the correct evidence. On the other hand, using the face clusters is vulnerable to errors since the wide variability in face appearance - especially the changes in pose, illumination conditions, facial expressions, and partial occlusions.

The facial features need to be informative and discriminative in order to distinguish the faces of different persons. Our alignment method using a likelihood function based on the feature vector $L^{N B}$ did a good job in optimizing the facial features and in taking into account the individual features of the faces and, as a result, it performs really well (see figure 7). Moreover, incorporating the clothing information into the face classification process is indeed helpful in the Buffy dataset. In the videos where people usually wear beautiful and color-distinguished clothing, utilizing these distinguished colors is advantageous.

The quality of the active learning for face selection in the user feedback setting also affects the final face labeling results. As discussed in section 5.1.3, the labels of the selected faces should be uncertain enough so that they would need the users help. And the set of the selected faces should be diverse enough to cover a sufficient number of the names. In many cases in the results, the alignment performance does not increase proportionally to the increase of the number of labeled faces since the active learning process selected many faces that are already correctly labeled when using the unsupervised approaches. This is because the quality of the $P(f \mid n)$ probability distribution is not optimal and the proportion of the number of labeled faces over name classes is not balanced (the diversity of the faces is not sufficient). The root of these problems converges to the factors discussed in the previous paragraph.

In most of our experiment settings, the use of picturedness and namedness scores (likelihood type $L^{\left(n^{*}, f^{*}\right)}$ ) does not help in improving the final alignment accuracy, and even causes a reduction of the final accuracy. In other words, the picturedness and namedness information is not informative as all detected faces refer to actors in the play and are equally likely mentioned in the texts. As illustrated in figure 1 and seen in many more cases, many faces appear in a frame and they are mentioned in the text regardless of their position or size. As for the names, it is hard to tell which names are most likely to be 


\begin{tabular}{|l||l||l|}
\hline \multirow{2}{*||}{ Method } & \multicolumn{2}{c|}{ Accuracy } \\
& Episode 0502 & Episode 0505 \\
\hline Everingham et al. [2] & $68.2 \%$ & $69.2 \%$ \\
\hline $\begin{array}{l}L^{N B} \text { with no namedness and pictured- } \\
\text { ness }\end{array}$ & $75 \%$ & $78 \%$ \\
\hline
\end{tabular}

Table 3: Comparison of Everingham et al.'s [2] and our best names and faces alignment results on "Buffy the Vampire Slayer" video dataset.

\begin{tabular}{|l||l|}
\hline Method & Accuracy \\
\hline Berg et al. [12] with language information & $77 \%$ \\
\hline$L^{\left(n^{*}, f^{*}\right)}[15]$ with namedness and picturedness & $77 \pm 1.29 \%$ \\
\hline$L^{N B}$ with namedness and picturedness & $68.77 \pm 0.32 \%$ \\
\hline
\end{tabular}

Table 4: Comparison of Berg et al.'s and our best names and faces alignment results on "Labeled Faces in the Wild" image dataset.

shown in the frames because the syntactic and semantic textual clues which are valuable in the still image-captions [15] do not seem to be useful in order to distinguish pictured names from the ones not shown.

Hence, the lesson we learn from our experiments of labeling faces in soap videos is that in order to reach acceptable performance the faces should have sufficient correct candidate names, the facial features should be optimized and, if available, other information can help.

Comparison to state-of-the-art work on the same Buffy dataset, Everingham et al. [2] report the precisions of $68.2 \%$ and $69.2 \%$ when they label $100 \%$ of the faces (i.e. without refusal-to-predict) in episodes 0502 and 0505 , respectively. Our results are better with precisions respectively of $75 \%$ and $78 \%$ when we use the $L^{N B}$ likelihood type alignment method. Table 3 shows the comparison of the results.

Although our techniques focus on the names and faces alignment in video data, we can apply our best method (i.e., likelihood type $L^{N B}$ ) with the following tuning to adapt to the text-image data ("Labeled Faces in the Wild"): 1) Each face track contains only one face; 2) No clothing information is used; 3) The facial features are now the results of our 3D morphable face representation model [15]. After running our unsupervised, $L^{N B}$ type alignment processe using only facial appearances (OF) with namedness and picturedness information, we randomly select and evaluate 1000 faces from the "Labeled Faces in the Wild" dataset 2000 times. We obtain minimum and 
maximum accuracies of $64 \%$ and $74 \%$, respectively, and the average accuracy and standard deviation of $68.77 \%$ and $0.32 \%$. These are lower than the best result reported by Berg et al. of $77 \%$ when language information was used and 1000 faces were randomly selected and evaluated. The compared numbers are shown in table 4 . These results are also lower than our best previous results $(77 \pm 1.29 \%)$ on this dataset reported in [15]. We suspect that the 3D morphable model already optimizes the facial features in "Labeled Faces in the Wild" dataset, hence re-optimizing them by our the Naive Bayes classfier is not necessary. Moreover, we train the Naive Bayes classifier for naming person in the image with the weak supervision of the text. More specifically, the alignment method with likelihood type $L^{N B}$ first learns a Naive Bayes classifer for each face with the names it finds in the accompanying text. The more name candidates are found in the text, the noisier the training example becomes as each name mentioned is assigned to each face in this training example. On "Labeled Faces in the Wild" dataset, we report from [15] that there are on everage 1.97 person names and 1.32 faces in one image with standard deviations of 0.0095 and 0.0057 , respectively. We can see that these numbers are higher than the numbers in the Buffy dataset (see table 2 ). To see more clearly how noisy the training examples are, we measure the degree of training class noise as follows. The noise degree is estimated as the percentage of the incorrect name assignments to the detected faces in all training examples. In the "Labeled Faces in the Wild" dataset, we have a noise degree of $59.20 \%$, while in the two Buffy episodes, the noise degrees are $45.21 \%$ and $46.07 \%$, respectively. This can explain the worse alignment performance of the Naive Bayes based $L^{N B}$ model on the "Labeled Faces in the Wild" dataset. 

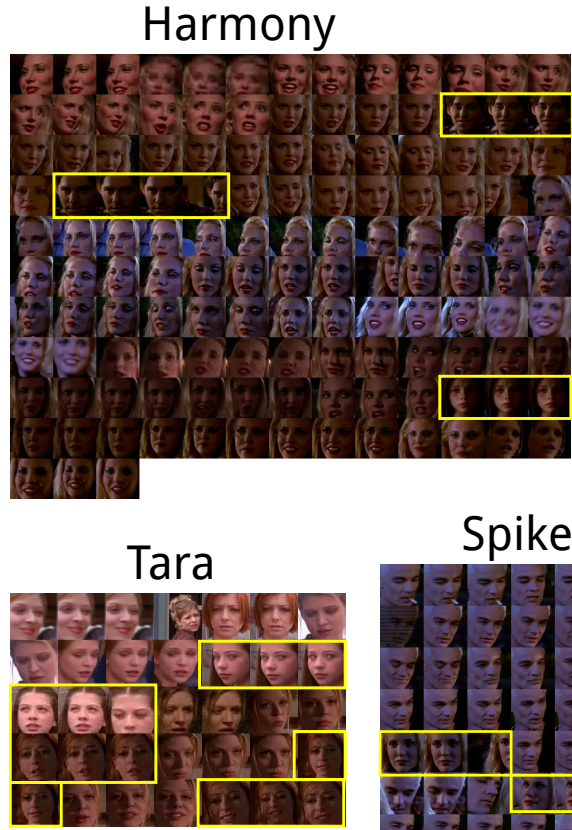

Giles

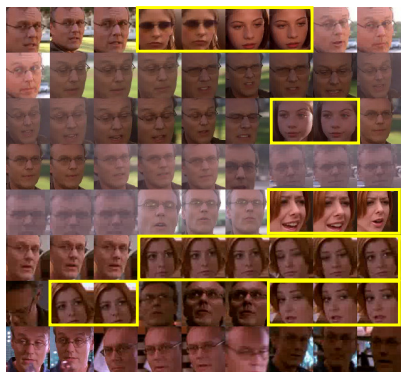

Riley

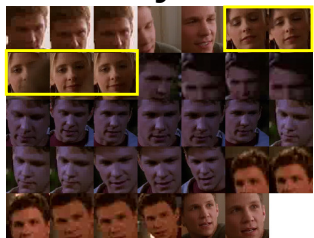

Dawn

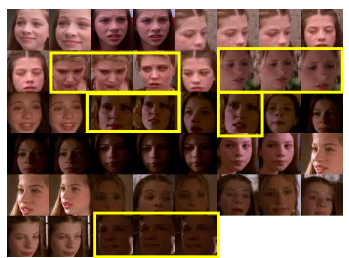

Xander

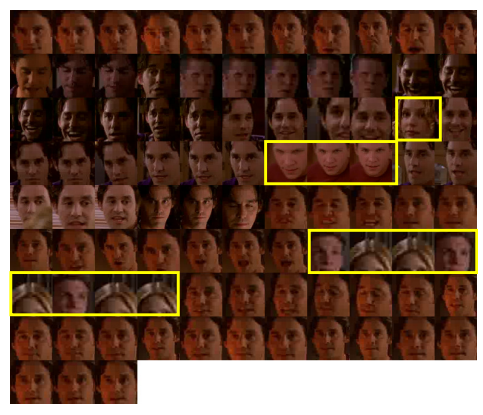

Willow

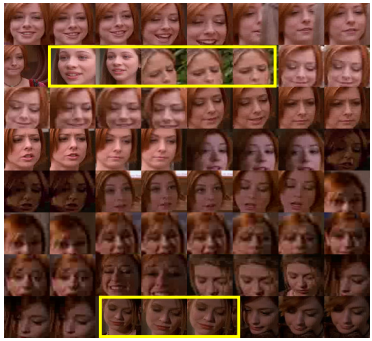

Buffy
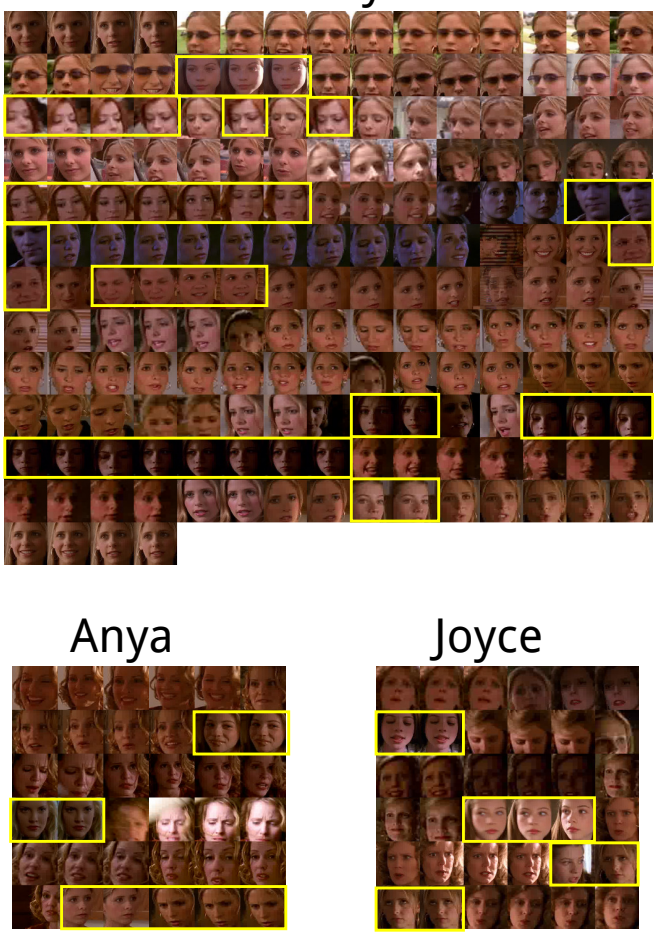

28

Figure 8: A representative example of face labeling results with the faces wrongly labeled shown in the yellow box. Note the variation of the lighting. (source: Buffy the Vampire Slayer episodes 05-02 and 05-05). 


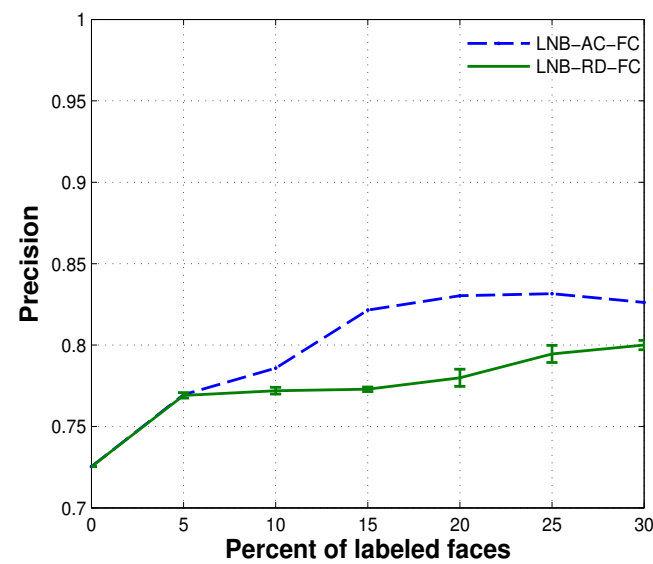

(a) Precision curves on episode 0502.

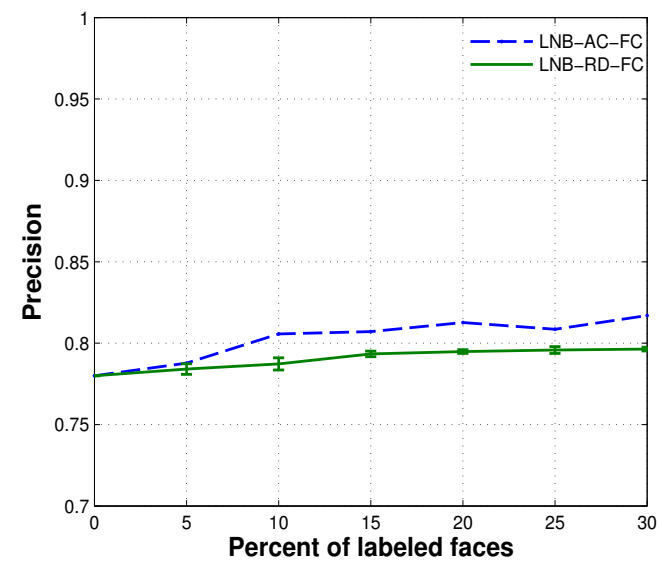

(c) Precision curves on episode 0505.

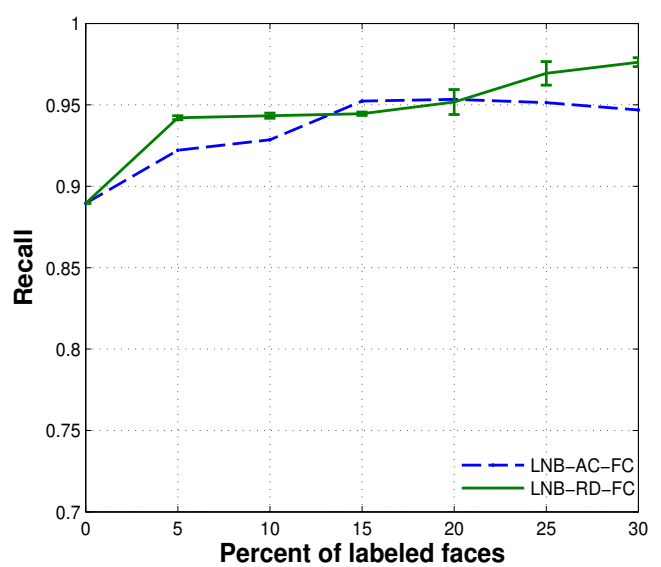

(b) Recall curves on episode 0502.

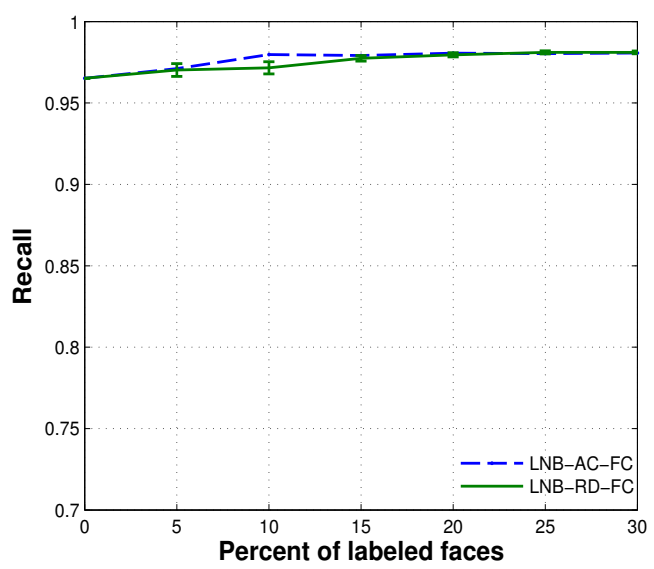

(d) Recall curves on episode 0505.

Figure 9: Precision/recall curves evaluated excluding the training faces of the alignment EM with likelihood function $L^{N B}$ using both facial and clothing appearance (FC). The experiments are run on the active labeled faces (AC) and the random labeled faces (RD). 


\section{Conclusion}

We have evaluated several face labeling algorithms for annotation of soap videos (Buffy the Vampire Slayer). In the first step based on the temporal alignment of the transcripts of fans, subtitles and video frames, mentioned names are loosely attributed to the corresponding frames in the video. Then an EM algorithm that exploits the redundant linking of a face and a name refines this name-face alignment. The results of the unsupervised approach are very competitive and reach $F 1$ values of $80 \%$ and $86 \%$, which are better than state-of-the-art results for face labeling obtained with the same dataset.

Our unsupervised face naming methods can easily be extended to deal with user feedback for labeling seed examples. We also introduce an active learning approach to effectively help the users to label example faces. The whole system covers important user aspects in the task of labeling faces in the videos: automatically labeling the faces and helping the user to provide feedback if he/she is not satisfied with the initial labeling results. Overall, the unsupervised face labeling methods have comparative performance with the supervised ones.

The techniques used in this study, although inspired by the alignment approach of [15], show some important improvements on the video data. The most important improvement is the Naive Bayes based alignment method which introduces the facial feature optimization in the learning-to-align process. Other add-on techniques to adapt to video data, such as face tracking and clothing detection, are also considered. More importantly, via our findings from the experiments, we recognize that some assumptions from text-image data are difficult to be applied in video data. For example, the assumption about the high possibility for a name to be depicted in the picture if this name is mentioned at the beginning of the sentence (see the experiments section) is in many cases not applicable for soap video data. Finding new or alternative assumptions for video data is an interesting direction for future research.

\section{References}

[1] P. T. Pham, T. Tuytelaars, M.-F. Moens, Naming people in news videos with label propagation, IEEE Multimedia 18 (2011) 44-55. doi:http://doi.ieeecomputersociety.org/10.1109/MMUL.2011.22. 
[2] M. Everingham, J. Sivic, A. Zisserman, Hello! My name is... Buffy - Automatic naming of characters in tv video, in: Proceedings of the British Machine Vision Conference, 2006.

[3] J. Yang, A. G. Hauptmann, Naming every individual in news video monologues, in: Proceedings of the ACM Multimedia 2004, 2004, pp. $580-587$.

[4] J. Yang, R. Yan, A. G. Hauptmann, Multiple instance learning for labeling faces in broadcasting news video, in: Multimedia '05: Proceedings of the 13th Annual ACM International Conference on Multimedia, ACM, 2005, pp. 31-40.

[5] D. Ramanan, S. Baker, S. Kakade, Leveraging archival video for building face datasets, in: Proceedings of the 2007 IEEE International Conference on Computer Vision, IEEE Computer Society, 2007, pp. 1-8. doi:http://doi.ieeecomputersociety.org/10.1109/ICCV.2007.4409012.

[6] S. Satoh, Y. Nakamura, T. Kanade, Name-it: Naming and detecting faces in news videos, IEEE Mutimedia 1 (1999) 22-35.

[7] D. Ozkan, P. Duygulu, A graph based approach for naming faces in news photos, in: CVPR '06: Proceedings of the 2006 IEEE Computer Society Conference on Computer Vision and Pattern Recognition, IEEE Computer Society, 2006, pp. 1477-1482. doi:http://dx.doi.org/10.1109/CVPR.2006.29.

[8] M. Guillaumin, T. Mensink, J. Verbeek, C. Schmid, Automatic face naming with caption-based supervision, in: Proceedings IEEE Conference on Computer Vision and Pattern Recognition, 2008.

[9] M. Guillaumin, T. Mensink, J. Verbeek, Improving people search using query expansions: How friends help to find people, in: European Conference on Computer Vision, 2008.

[10] L. Cao, J. Luo, T. S. Huang, Annotating photo collections by label propagation according to multiple similarity cues, in: Proceedings of the 16th ACM International Conference on Multimedia, ACM, New York, NY, USA, 2008, pp. 121-130. 
[11] K. Grauman, T. Darrell, Unsupervised learning of categories from sets of partially matching image features, in: Proceedings of the IEEE Conference on Computer Vision and Pattern Recognition (CVPR), 2006.

[12] T. L. Berg, A. C. Berg, J. Edwards, D. Forsyth, Who's in the picture, in: L. K. Saul, Y. Weiss, L. Bottou (Eds.), Advances in Neural Information Processing Systems 17, MIT Press, 2005, pp. 137-144.

[13] T. Berg, A. Berg, J. Edwards, M. Maire, R. White, Y. Teh, E. LearnedMiller, D. Forsyth, Names and faces in the news, in: Proceedings of the 2004 IEEE Conference on Computer Vision and Pattern Recognition, Vol. 2, 2004, pp. 848-854.

[14] P. Duygulu, K. Barnard, N. de Freitas, D. Forsyth, Object recognition as machine translation: Learning a lexicon for a fixed image vocabulary, in: Proceedings of the Seventh European Conference on Computer Vision, 2002, pp. 97-112.

[15] P. T. Pham, M.-F. Moens, T. Tuytelaars, Cross media alignment of names and faces, IEEE Transactions on Mutimedia 12 (1) (2010) 13-27.

[16] A. Tian, M. Lease, Active learning to maximize accuracy vs. effort in interactive information retrieval, in: Proceedings of the 34th international ACM SIGIR conference on Research and development in Information, SIGIR '11, ACM, New York, NY, USA, 2011, pp. 145-154. doi:http://doi.acm.org/10.1145/2009916.2009939. URL http://doi.acm.org/10.1145/2009916.2009939

[17] T. Huang, C. Dagli, S. Rajaram, E. Chang, M. Mandel, G. Poliner, D. Ellis, Active learning for interactive multimedia retrieval, Proceedings of the IEEE 96 (4) (2008) 648 -667. doi:10.1109/JPROC.2008.916364.

[18] P. Viola, M. Jones, Robust realtime object detection vector quantization, International Journal of Computer Vision 57 (2) (2004) 137-154.

[19] D. Lowe, Object recognition from local scale-invariant features, in: The Proceedings of the Seventh IEEE International Conference on Computer Vision, Vol. 2, IEEE, 1999, pp. 1150-1157. 
[20] J. Shi, C. Tomasi, Good features to track, in: Proceedings of the Conference on Computer Vision and Pattern Recognition, IEEE Computer Society Press, 1994, pp. 593-600.

[21] Y. Mori, H. Takahashi, R. Oka, Automatic words assignment to images based on image division and vector quantization, in: Proceedings of RIAO' 2000 Content-Based Multimedia Information Access, 2000.

[22] K. Deschacht, M.-F. Moens, Text analysis for automatic image annotation, in: Proceedings of the 45th Annual Meeting of the Association of Computational Linguistics, ACL, Prague, Czech Republic, 2007, pp. 1000-1007.

URL http://www . aclweb.org/anthology/P/P07/P07-0126

[23] E. Charniak, A maximum entropy-inspired parser, in: Proceedings of the First Conference of the North American Chapter of the Association for Computational Linguistics, 2000. 